\title{
Make maps great again
}

\author{
Alejandro Guinea de Salas ${ }^{\text {a }}$ \\ ${ }^{a}$ Geograma, alejandro.guinea@geograma.com
}

Keywords: WMS, OGC, quality control, quality assurance, Maps, style, symbology, legend

\begin{abstract}
:
Only few years ago, maps and paper were directly related. A map and the paper containing the map were the same thing. The map was the paper, and the paper was the map. Even at the beginning of this century, the main GIS and CAD outputs were plots. Each a every cartography company had a plotter, because the deliverables of the usual projects were sheets of paper containing maps. Those maps had two parts, the frame and the content itself. They were the most powerful way to see the territory from a global point of view.
\end{abstract}

Digitalisation affected the maps industry like a tsunami, like everywhere else. The first step was to convert the paper into PDFs. The material was complete changed, paper vanished, but the main static content and tools were nearly the same.

But after the creation of the OGC protocols, the disruption of google maps, and the implementation of the INPSIRE Directive, a new way of maps appeared in the scene. Web Map Service (WMS) protocol allowed to share maps in a completely different way. Not only the format, but the infrastructure needed, and the content suffered a massive disruption. The user of the maps was able to access huge quantity of data, from their pc or mobile, connecting directly in real time to the data provider, at many different zooms, to the last up to date information. We went from "The Map is in internet" to "the map is internet".

However, at the same time, something went a step back. Texts vanished from maps, the frame of the map with crucial information disappeared (who made it, when, legend, scale, date). Colours and styles were simplified so much, scale became a strange concept. It was like all the tools to help how to interpret the map were delegated in the user's skills to navigate and configure the WMS at his own. Any cartographer will understand how far the maps are nowadays from the paper classic maps.

In this article, we will share best practices to make the maps served though WMS the best tool to communicate information about the territory. What to do with texts, how to proceed with layers, where to put the relevant information, and how important is to have readable legends.

We will share examples of public institutions, to show how the huge value, and cost, of the geographic information can be improved. 